\title{
Reconhecimento de Emoções em Expressões Faciais: Estudo Exploratório Envolvendo Adultos
}

\author{
Rodolfo de Castro Ribas Junior* \\ Universidade Federal do Rio de Janeiro, Rio de Janeiro, Brasil \\ Marcos de Souza Aguiar \\ Universidade Federal Rural do Rio de Janeiro, Rio de Janeiro, Brasil \\ Paula Bandeira Dias, Felipe Salvador Grisolia, Luiz Gonzaga Ribeiro Vasconcelos Rosario,
Felipe Costa Pulcherio Lima \& Paulo Koatz Miragaya
Universidade Federal do Rio de Janeiro, Rio de Janeiro, Brasil
}

\begin{abstract}
RESUMO
O presente trabalho relata a realização de um estudo exploratório sobre o reconhecimento de emoções em expressões faciais (REEF) em uma amostra de adultos brasileiros. Participaram do estudo 118 estudantes universitários. Foram utilizados dois instrumentos para a avaliação do reconhecimento de emoções: O DANVA2, um instrumento amplamente utilizado na literatura internacional e um segundo instrumento desenvolvido pelos autores (EREEF). Os participantes completaram ainda escalas para avaliação sintomatologia para depressão e de bem-estar subjetivo e qualidade de interação com os pais. Corroborando estudos anteriores, participantes do sexo feminino apresentaram maior capacidade de reconhecimento de emoções em expressões faciais quando comparados a participantes do sexo masculino. Esses dois grupos não diferiram significativamente em nenhuma das outras medidas estudadas. Não foram identificadas correlações significativas entre as medidas de reconhecimento de emoções e as medidas de sintomatologia para depressão e de bem-estar subjetivo e qualidade de interação com os pais.
\end{abstract}

Palavras-chave: reconhecimento de emoções; expressões faciais; emoções.

\section{ABSTRACT \\ Emotion Recognition in Facial Expressions: An Exploratory Study Involving Adults}

The present work describes an exploratory study on in facial expressions in a sample of Brazilian adults. Participants were 118 college students. Two instruments were used for the assessment of emotion recognition: the DANVA2, an instrument largely employed in the international literature and a second instrument developed by the authors (EREEF). Participants also completed scales to assess symptoms of depression, subjective well-being, and quality of interaction with parents. Corroborating previous investigations, female participants presented higher competence on emotions' recognition in facial expressions when compared to male participants. These two groups did not differ significantly in any of the other measures studied. No significant correlations were identified between measures of emotion recognition and symptoms for depression, subjective well-being, and quality of interaction with parents.

Keywords: emotion recognition, facial expressions, emotions.

Artigos de revisão extensos e relativamente recentes têm apontado que o número de pesquisas sobre as emoções humanas e tópicos relacionados tem crescido substancialmente nas últimas duas décadas (e.g., Mayer, Roberts \& Barsade, 2008). De fato, investigadores têm afirmado que, na sequência da tão comentada revolução cognitivista (Robins, Gosling, \& Craik, 1999), estaria em curso uma "revolução afetiva" ou uma "revolução da emoção". Weber e Johnson (2009, p. 65), por exemplo, mencionam explicitamente o andamento de uma "emotions revolution". Nessa revolução, a ênfase, talvez excessiva, dada a componen-

*Endereço para correspondência: Rodoldo de Castro Ribas Junior - rodolforibas@ globo.com 
Rodolfo de Castro Ribas Junior, Marcos de Souza Aguiar, Paula Bandeira Dias, Felipe Salvador Grisolia,

Luiz Gonzaga Ribeiro Vasconcelos Rosario, Felipe Costa Pulcherio Lima \& Paulo Koatz Miragaya

tes cognitivos nas pesquisas psicológicas estaria sendo reavaliada e a real importância de componentes emocionais em processos como resolução de problemas e tomada de decisão estaria sendo cada vez mais reconhecida. De fato, além do crescimento de pesquisas com foco nos processos afetivos, pode-se também dizer cresce o número de investigações que analisam simultaneamente variáveis cognitivas e afetivas (Izard, 2009; Southam-Gerow \& Kendall, 2002).

As pesquisas sobre emoções tem focalizado uma ampla gama de tópicos como temperamento, bases neurais das emoções, regulação das emoções e inteligência emocional (e.g., Izard, 2001; Hoehl \& Striano, 2008; Mayer, Salovey, \& Caruso, 2008;). Entre os temas investigados podemos citar o reconhecimento de emoções. Southam-Gerow e Kendall (2002), por exemplo, examinaram a importância da compreensão das emoções no ajustamento psicológico. A compreensão das emoções envolveria basicamente dois componentes: 1) a capacidade de reconhecer emoções em expressões faciais, posturas corporais, tons de voz e outras formas humanas de expressão (e.g., arte) e 2) conhecimentos sobre as emoções humanas. Segundo esses autores, o conhecimento das emoções poderia ser ainda divido em cinco subcomponentes: a) as causas das emoções em nós e nos outros, b) as pistas sobre as emoções em nós e nos outros, c) a possibilidade de sentir diferentes emoções acerca de um mesmo objeto, d) formas de comunicar ou ocultar intencionalmente uma emoção, e e) formas de lidar de maneira efetiva com as emoções (Southam-Gerow\& Kendall, 2002, p. 200). De uma maneira geral, pode-se dizer que indivíduos com mais capacidade de reconhecer emoções tenderiam a apresentar um melhor ajustamento emocional.

A capacidade de identificar emoções em faces, vozes, posturas e outros conteúdos também tem sido identificada como um importante componente da inteligência emocional (e.g., Mayer, Roberts, \& Barsade, 2008; Mayer, Salovey \& Caruso, 2008). Entre os outros componentes normalmente citados dessa forma de inteligência, poderíamos mencionar: utilizar as emoções para raciocinar e solucionar problemas mais efetivamente, lidar de forma eficaz com respostas emocionais, e entender as emoções, seu curso e significados. Nesse contexto, pessoas com maior capacidade de reconhecer corretamente emoções tenderiam a ter uma maior inteligência emocional, uma habilidade humana que, por definição, envolveria relações entre processos afetivos e cognitivos.
A presente investigação focaliza exatamente o reconhecimento de emoções em faces humanas. Diversos estudos têm indicado que a capacidade de reconhecer emoções humanas em expressões faciais é uma importante habilidade que seres humanos utilizam no seu dia a dia, em interações sociais, e um importante componente envolvido em processos como regulação emocional, resolução de problemas e liderança.

Estudos envolvendo a expressão e o reconhecimento de emoções em faces tem uma longa história (e.g., Darwin, 1872/2002). Todavia, pode-se dizer que os estudos nessa área foram grandemente impulsionados pelas contribuições de Paul Ekman e colaboradores (e.g., Ekman, 1993, 2012; Ekman \& Cordaro, 2011; Ekman \& Friesen, 1971; Ekman \& Oster, 1979). Os estudos de Ekmancertamente ampliaram nosso conhecimento sobre como as emoções humanas são reconhecidas. Ekman e Cordaro (2011), apenas para citar um exemplo, defenderam, em uma recente revisão, que há evidências claras acerca da existência de sete emoções básicas universais: raiva, medo, surpresa, tristeza, repugnância, menosprezo e felicidade.

Ainda no que se refere ao reconhecimento de emoções em faces, um outro pesquisador precisa ser mencionado: Stephen Nowicki Jr. Nowicki e colaboradores (e.g., Nowicki \& Carton, 1993, 1997, Nowicki \& Duke, 1994, Nowicki, Glanville \& Demertzis, 1998, Mufson \& Nowicki, 1991) têm continuamente desenvolvido estudos sobre o reconhecimento de emoções em faces, vozes e posturas. Nowicki e Duke (1994), por exemplo, desenvolveram um dos instrumentos que mais frequentemente tem sido hoje utilizado a nível internacional para medir a capacidade de reconhecimento de emoções em faces, a Diagnostic Analysis of Nonverbal Accuracy Scale (DANVA2). De fato, em julho de 2013 nada menos que 40 artigos científicos mencionavam a utilização de alguma versão do DANVA. Na presente pesquisa foi empregado a DANVA2, que foi cedida por seu idealizador, Stephen Nowicki Jr e um novo instrumento desenvolvido pelos autores com a mesma finalidade. Nesse sentido, pode-se dizer que os autores privilegiaram a perspectiva desenvolvida Nowicki Jr e seus colaboradores nesta investigação. O DANVA2 avalia quatro emoções básicas (alegria, tristeza, raiva e medo).

Apesar da capacidade de reconhecimento de emoções em facesser uma importante habilidade humana, foi possível verificar, por meio de uma ampla e sistemática revisão da literatura, a ausência quase que total de publicações e estudos empíricos nacionais sobre o assunto. Desta forma, considera-se que o presente 
estudo pode contribuir para a redução dessa lacuna, ao fornecer evidências obtidas em uma investigação envolvendo participantes brasileiros.

\section{Reconhecimento de emoções em expressões faciais: evidências empíricas mais recentes}

De uma maneira geral, pode-se dizer que a relevância de componentes neurológicos, maturacionais, ambientais, sociais e culturais têm sido identificada. De fato, a maior parte dos estudos contemporâneos integram estes diferentes componentes entre si em modelos interacionistas (e.g., McClure, 2000, Vigil, 2000).

Montirosso, Peverelli, Frigerio, Crespi e Borgatti (2010) estudaram o desenvolvimento do reconhecimento de expressões faciais (alegria, raiva, medo, nojo e tristeza) em um grupo de 240 crianças e jovens italianos com idades entre 04 e 18 anos de idade. Foram utilizadas uma escala de inteligência padronizada e uma tarefa computadorizada com expressões faciais animadas (AFFECT, Animated Full Facial Expression Comprehension Test). Os resultados indicaram que a capacidade de reconhecimento de expressões aumenta ao longo da infância e da juventude, com exceção do reconhecimento do nojo. Verificou-se que a capacidade de perceber o nojo é uma das primeiras a ser aprendida, não variando entre 04 e 18 anos. Observou-se ainda que participantes do sexo feminino apresentaram melhor desempenho que participantes do sexo masculino, principalmente no que diz respeito ao reconhecimento de expressões de nojo e raiva. Beek e Dubas (2008) investigaram o reconhecimento de expressões faciais em um grupo de 606 crianças e jovens holandeses, com idades entre 09 e 15 anos e também verificaram que a capacidade de reconhecer expressões emocionais, especialmente as de baixa intensidade, aumenta com a idade. Nesse estudo foram apresentadas expressões faciais sob a forma de desenhos.

Pesquisas têm sistemática e consistentemente indicado que crianças e adolescentes do sexo feminino apresentam uma maior habilidade para identificar emoções em expressões faciais que crianças e adolescentes do sexo masculino. McClure (2000), por exemplo, conduziu uma meta-análise com mais de uma centena de estudos e os resultados foram convergentes. Os dados agregados identificaram um tamanho do efeito corrigido $(D+)$ igual a 0,16 , favorecendo crianças e adolescentes do sexo feminino que significativamente obtiveram escores maiores que crianças e adolescentes do sexo masculino. Kring e Gordon (1998) contribuíram para a compreensão desse achado ao identificarem que não apenas o sexo, mas também o gênero (inferido por uma escala de masculinidade e feminilidade) e o ambiente familiar podem estar associados a essa diferença. No presente estudo essa questão também foi examinada. A capacidade de reconhecimento de homens e mulheres foi comparada.

Estudos sobre o reconhecimento de emoções em expressões faciais têm ainda identificado múltiplas associações entre essa competência, o ambiente familiar, o ajustamento emocional e outras variáveis psicológicas. De fato, a habilidade de reconhecer emoções parece ser influenciada, entre outros aspectos, pelo contexto familiar e tem papel relevante no processo de desenvolvimento humano, tanto normal quanto atípico (e.g., Propper \& Moore, 2006). Pears e Fisher (2005), por exemplo, ponderam que evidências têm indicado que crianças submetidas a maus-tratos e crianças com transtornos psicológicos tendem a apresentar prejuízos na capacidade de reconhecer emoções. No presente estudo foi incluída uma medida de qualidade de interação entre pais e filhos e filhas. Buscou-se avaliar a influência dessa variável na capacidade de perceber emoções.

Convergindo com os resultados de pesquisa reportados até aqui, Izard et al. (2001) verificaram que a habilidade para reconhecer e nomear expressões emocionais foi um preditor de longo prazo de comportamento social positivo e de competência acadêmica em crianças (ver também Cervantes \& Callanan, 1998). Goodfellow e Nowicki (2009) estudaram a habilidade de reconhecer expressões faciais e competência social e acadêmica em um grupo de 840 crianças com idades entre 7 e 10 anos. Estes autores concluíram que crianças que apresentaram maior habilidade para a identificação de emoções tendem a apresentar maior competência social e acadêmica.

McClure e Nowicki (2001) investigaram uma possível associação entre a ansiedade social e a capacidade de crianças para decodificar sinais não verbais de emoções (i.e., expressões faciais e posturas corporais). Observou-se que crianças mais hábeis para identificar emoções apresentaram menor ansiedade social. Para McClure e Nowicki (2001) esses resultados sugerem que a ansiedade social pode estar ligada a uma disfunção no processamento da informação social nas fases iniciais do desenvolvimento. 
Rodolfo de Castro Ribas Junior, Marcos de Souza Aguiar, Paula Bandeira Dias, Felipe Salvador Grisolia,

Luiz Gonzaga Ribeiro Vasconcelos Rosario, Felipe Costa Pulcherio Lima \& Paulo Koatz Miragaya

Num estudo envolvendo adultos, Carton, Kessler e Pape (1999) identificaram inter-relações entre bem-estar subjetivo avaliado a partir da Ryff Psychological Well-Being Scale, sintomas de depressão avaliados através da Escala de Sintomas de Depressão CES-D e reconhecimento de emoções faciais. Os autores identificaram uma correlação positiva entre reconhecimento de emoções e bem-estar e uma correlação negativa entre reconhecimento de emoções e quantidade de sintomas de depressão. A relação entre essas três variáveis foi explorada no presente estudo.

Uma revisão sistemática da literatura nacional foi conduzida. Apenas dois estudos empíricos sobre o reconhecimento de emoções em expressões faciais conduzidos no Brasil foram identificados. Alves-Neto et al. (2010) testaram o efeito dos níveis de serotonina no desempenho em uma tarefa de reconhecimento de emoções faciais (raiva, nojo, medo, alegria, tristeza e surpresa) em uma amostra de 12 adultos brasileiros. Os autores reportaram que níveis mais baixos de serotonina facilitaram a percepção de tristeza e dificultaram a percepção de alegria. $\mathrm{O}$ estudo parece indicar a existência de relações entre o estado emocional e o reconhecimento de emoções. No presente estudo essa questão foi examinada. Foram analisadas correlações entre medidas de estado emocional (sintomas de depressão e bem-estar subjetivo) e a habilidade de reconhecer emoções.

Neves et al. (2011) investigaram a percepção de emoções faciais em um grupo de 81 pais residentes em Minas Gerais. Aproximadamente metade desses pais (40) possuía um filho portador de autismo, enquanto que a outra metade (41) possuía filhos saudáveis. Entre outros objetivos, a pesquisa testou o efeito de um gene (5HTTLPR) na percepção de emoções. O estudo não identificou associações entre a presença do genótipo para o 5HTTLPR e o desempenho no teste de reconhecimento de emoções. Entretanto verificou-se que pais de crianças portadores de autismo apresentaram pior reconhecimento de emoções faciais quando comparados ao grupo de controle.

O presente trabalho relata a realização de um estudo exploratório sobre o reconhecimento de emoções em expressões faciais em uma amostra de adultos brasileiros. Um instrumento de medida internacional e outro desenvolvido pelos autores foram utilizados para avaliar essa capacidade. No estudo foram examinadas prioritariamente as relações entre sexo, estado emocional, histórico de interação com os pais e a capacidade de perceber emoções.

\section{MÉTODO}

\section{Participantes}

Participaram desta pesquisa 118 estudantes de um curso de psicologia situado no Rio de Janeiro, sendo $94(80,3 \%)$ do sexo feminino e $23(19,7 \%)$ do sexo masculino (1 participante não forneceu essa informação). A proporção de estudantes do sexo masculino na amostra foi compatível com a proporção de estudantes desse sexo com matrícula ativa no curso de bacharelado em psicologia $(22,1 \%$ de 946 estudantes com matrícula ativa). A idade dos estudantes oscilou entre 18 e 34 anos, $(M=20,4$; DP = 2,71).

\section{Instrumentos}

Termo de Consentimento Livre e Esclarecido (TCLE). Os participantes tomaram conhecimento de informações básicas sobre a pesquisa (objetivos, ris$\cos$ e benefícios, confidencialidade) por meio do TCLE. Este termo foi previamente aprovado por um Comitê de Ética em Pesquisa.

Reconhecimento de Emoções em Expressões $\mathrm{Fa}$ ciais. A Escala de Reconhecimento de Expressões Faciais (EREEF) foi utilizada no estudo. A EREEF é composta por 28 fotografias que apresentam expressões faciais de quatro emoções (alegria, tristeza, raiva e medo) com diferentes níveis de intensidade, sendo as quatro primeiras fotos utilizadas como pré-teste para familiarização com o procedimento. Os resultados dessas fotos não foram analisados.

$\mathrm{O}$ instrumento foi desenvolvido pelos autores com base em outra escala, a Diagnostic Analysis of Nonverbal Accuracy Scale (DANVA2), originalmente concebida por Stephen Nowicki Jr. et al. (e.g., Nowicki \& Duke, 1994). Na versão empregada neste estudo as fotos são apresentadas em um computador acoplado em um projetor (datashow). Cada fotografia é apresentada por 2 segundos, sendo aplicado um intervalo de 5 segundos entre as fotos. Foi solicitado aos participantes que identificassem a emoção representada em cada fotografia. Escores (percentagem de respostas corretas) foram computados para o resultado geral e para o resultado obtido em cada emoção. Percentagens maiores indicam maior habilidade para reconhecer emoções. No presente estudo foi observado um alfa de Cronbach de 0,86, indicando adequada consistência interna.

O DANVA2 (Nowicki \& Duke, 1994) também foi empregado no estudo, servindo como modelo inspirador e critério de validação da EREEF. Esse teste com- 
putadorizado é composto por 24 fotografias de expressões faciais (alegria, tristeza, raiva e medo) de diferentes intensidades (alta e baixa), em número igual e com um tempo de exposição de 2 segundos para cada foto. Escores (percentagem de respostas corretas) foram computados para o resultado geral e para o resultado obtido em cada emoção. Percentagens maiores indicam maior habilidade para reconhecer emoções. Este instrumento vem sendo utilizado em dezenas de pesquisas ao redor do mundo para medir essa competência em adultos e crianças (Mayer, Roberts \& Barsade, 2008). O autor do teste disponibilizou o instrumento para utilização no estudo brasileiro. A consistência interna do DANVA2 tem sido satisfatória. A maioria dos estudos (e.g., Nowicki \& Carton, 1997) tem reportado alfas de Cronbach em torno de 0,77 em adultos. No presente estudo foi utilizada uma versão adaptada para aplicação em grupo, sendo observado um alfa de Cronbach de 0,73, indicando adequada consistência interna.

Sintomas de depressão. Foi utilizada uma versão em português da Escala de Sintomas de Depressão CES-D (Radloff, 1977; Center for Epidemiologic Studies - Depression Scale). Esta escala tem como objetivo avaliar a ocorrência de sintomatologia relacionada a estados depressivos na população de uma maneira geral. O questionário é composto por 20 itens (Ex. Eu me senti triste.). As respostas são dadas em uma escala de quatro níveis (Raramente ou Nunca, menos que 1 dia; Muito pouco, 1 - 2 dias; Ocasionalmente, $3-4$ dias; Frequentemente, 5-7 dias), de acordo com a frequência com que o participante avalia que teve um determinado sintoma na semana anterior. Escores maiores indicam maior presença de sintomas de depressão. Estudos tem tipicamente relatado alfas em torno de 0,89 (e.g., Wong, 2000). No presente estudo foi observado um alfa de Cronbach de 0,84, indicando adequada consistência interna da versão em português dessa escala.

Bem-Estar Subjetivo. Versões em português do Índice de Satisfação com a Vida (Life Satisfaction Index, LSA) e da Escala de Afetos Positivos e Afetos Negativos (Positive Affect/Negative Affect Scale, PANAS), validadas inicialmente por Watson, Clark e Tellegen (1988) foram utilizadas para avaliar o bemestar subjetivo. O LSA é composto de 15 itens (e.g., Quando olho para minha vida, eu me sinto muito satisfeito(a)) enquanto que a PANAS é composta de 20 itens (e.g., Sinto-me tenso(a)). Watson, Clark e Tellegen (1988) reportaram alfas entre 0,86 e 0,90 para as escalas em referência. Escores maiores indicam uma maior satisfação com a vida e uma maior presença de afetos positivos acompanhada de menor presença de afetos negativos. No presente estudo os alfas observados foram satisfatórios, 0,89 e 0,82 para o LSA e a PANAS, respectivamente.

Qualidade do vínculo com os pais. Para avaliar a percepção dos participantes sobre qualidade do vínculo que eles tiveram com seus pais ou cuidadores, foi utilizada uma versão em português do Parental Bonding Instrument (PBI). Esse instrumento é constituído de 25 questões. Os participantes foram solicitados a lembrar determinadas atitudes e comportamentos apresentados por seus pais e mães até seus 16 anos (e.g., Parecia compreender meus problemas e preocupações.). Para cada afirmação eles deveriam escolher entre as seguintes opções: muito parecido, um pouco parecido, um pouco diferente, muito diferente. Uma versão em português do PBI foi desenvolvida por Hauck et al. (2006). Os participantes responderam uma versão do questionário referente ao comportamento de suas mães e uma referente ao comportamento de seus pais. No presente estudo os alfas foram satisfatórios e oscilaram entre 0,87 e 0,88 .

\section{Procedimentos}

Primeiramente os participantes preencheram um Termo de Consentimento Livre e Esclarecido. Na sequência, a Escala de Reconhecimento de Expressões Faciais (EREEF) e o DANVA2 (Diagnostic Analysis of Nonverbal Accuracy Scale - 2 Módulo Faces) foram aplicados coletivamente com o uso de computadores e projetores. Finalmente, foram respondidos individualmente a Escala de Sintomas de Depressão (CES-D), o Índice de Satisfação com a Vida (LSA), a Escala de Afetos Positivos e Afetos Negativos (PANAS) e o Parental Bonding Instrument (PBI).

\section{RESULTADOS}

Os participantes do estudo identificaram corretamente 74,2\% (DP = 19,9) das emoções apresentadas nas fotografias da Escala de Reconhecimento de Expressões Faciais (EREEF) e 70,7\% (DP = 14,2) das emoções apresentadas na versão adaptada do Diagnostic Analysis of Nonverbal Accuracy Scale (DANVA2). Os escores observados com a aplicação do DANVA2 são compatíveis com resultados reportados em estudo anteriores. Wickline, Bailey e Nowicki (2009), por exemplo, reportaram percentagens de acerto entre $63,8 \%$ e $76,7 \%$ em amostras de adultos. Os resultados 
obtidos com a Escala de Sintomas de Depressão do CES (CES-D), o Índice de Satisfação com a Vida (LSA) e a Escala de Afetos Positivos e Afetos Negativos (PANAS) também foram compatíveis com os resultados usualmente repostados na literatura. Por exemplo, Gonçalves e Fagulha (2004) reportaram médias entre 13,9 e 20,1 em uma aplicação de uma versão portuguesa da CES-D. No presente estudo a média observada para essa escala foi 18,63 (DP = 8,62). As médias observadas para a LSA, PANAS, PBI Comportamento Materno e PBI Comportamento Paterno foram, respectivamente: 3,44 ( $\mathrm{DP}=0,74), 3,66$ $(\mathrm{DP}=0,53), 3,04(\mathrm{DP}=0,46), 2,96(\mathrm{DP}=0,53)$.
A Tabela 1 apresenta comparações entre médias obtidas por participantes do sexo masculino e do sexo feminino em todas as principais medidas aplicadas no estudo. Como se pode observar, as mulheres apresentaram médias significativamente superiores às obtidas pelos homens nas duas medidas de reconhecimento de emoções em expressões faciais (EREEF e DANVA2). Esses dois grupos não diferiram significativamente em nenhuma das outras três medidas estudadas (CES-D, LSA, PANAS, PBI Materno e PBI Paterno). Esses resultados são compatíveis com a literatura internacional. McClure (2000), por exemplo, ressaltou que grande parte das pesquisas tem apontado que as mulheres apresentem melhor desempenho que homens em reconhecimento de emoções em expressões faciais.

Tabela 1

Escores obtidos com a aplicação das escalas considerando sexo dos participantes

\begin{tabular}{|c|c|c|c|c|c|}
\hline \multicolumn{6}{|c|}{ Sexo } \\
\hline Teste & Feminino & Masculino & $t$ & & $D$ \\
\hline EREEF & $76,64(17,57)$ & $65,22(25,95)$ & 2,52 & ** & 0,59 \\
\hline DANVA2 & $72,43(11,87)$ & $64,13(20,48)$ & 2,56 & $* *$ & 0,60 \\
\hline CES-D & $18,58(8,64)$ & $18,14(8,24)$ & 0,22 & & 0,05 \\
\hline LSA & $3,46(0,75)$ & $3,40(0,67)$ & 0,35 & & 0,08 \\
\hline PANAS & $3,64(0,53)$ & $3,85(0,41)$ & $-1,80$ & & $-0,43$ \\
\hline PBIMAT & $3,04(0,48)$ & $3,06(0,41)$ & $-0,18$ & & $-0,04$ \\
\hline PBIPAT & $2,94(0,53)$ & $3,07(0,49)$ & $-1,02$ & & 0,25 \\
\hline
\end{tabular}

Média (Desvio Padrão). EREEF - A Escala de Reconhecimento de Expressões Faciais; DANVA2 - Diagnostic Analysis of Nonverbal Accuracy Scale; CES-D - Escala de Sintomas de Depressão do CES; LSA - Índice de Satisfação com a Vida; PANAS - Escala de Afetos Positivos e Afetos Negativos. . PBIMAT Parental Bonding Instrument - Materno, PBIPAT Parental Bonding Instrument - Paterno.

Para avaliar se a percentagem de acertos variou considerando os quatro tipos de emoção apresentadas nas fotografias (alegria, tristeza, raiva e medo) foi realizada uma análise de variância para medidas repetidas (modelo GLM) com tipo de emoção (4) como fator intrasujeitos, para cada uma das medidas de reconhecimento de emoção (EREEF, DANVA2). Essas análises revelaram que a percentagem de acertos variou significativamente considerando a emoção, tanto no EREEF quanto no DANVA2, Wilks' Lambda $F=(3$, $115)=19,36, \mathrm{p}<0,001 ; F=(3,153)=36,25, \mathrm{p}<$ 0,001 , respectivamente. A Tabela 2 apresenta as mé- dias e desvios considerando emoção e escala. Comparações Post Hoc revelaram similaridades e pequenas variações entre o EREEF e o DANVA2. Tanto no EREEF quanto no DANVA2 os participantes identificaram mais facilmente expressões de alegria. Entretanto, no EREEF apenas a percentagem de acertos relacionada à alegria foi significativamente maior que as percentagens observadas nas outras emoções. No DANVA2 a percentagem de acertos relacionada a medo também foi significativamente maior que a percentagem de acertos para tristeza e raiva. 
Tabela 2

Percentagem de reconhecimento considerando tipo de emoção

\begin{tabular}{llllll}
\hline Teste & Alegria & Raiva & Tristeza & Medo & F \\
\hline EREEF & $84,07(21,81)$ & $72,03(25,70)$ & $71,53(27,10)$ & $71,43(25,95)$ & $19,38^{* *}$ \\
DANVA2 & $83,05(20,67)$ & $60,59(20,75)$ & $65,40(22,66)$ & $73,73(22,69)$ & $36,25^{* *}$ \\
\hline $\begin{array}{l}\text { Média (Desvio Padrão). EREEF - A Escala de Reconhecimento de Expressões Faciais, DANVA2 - Diagnostic Analysis } \\
\text { of Nonverbal Accuracy Scale. }\end{array}$
\end{tabular}

Finalmente, foram conduzidas de análise de correlação de Pearson com o objetivo de identificar correlações entre as variáveis investigadas. A Tabela 3 apresenta a matriz de correlações obtida. Diversas correlações significativas dignas de nota firam identificadas. Verificou-se, por exemplo, que as duas medi- das de reconhecimento de emoções, a EREEF e a DANVA2 apresentaram correlação significativa de média intensidade. De fato, essas duas medidas compartilharam aproximadamente $27 \%$ de suas variâncias. Esse resultado indica que esses instrumentos, de fato, mediram uma mesma dimensão.

Tabela 3

Correlações (Pearson) de ordem zero entre variáveis

\begin{tabular}{|c|c|c|c|c|c|c|c|c|c|}
\hline & 1 & 2 & 3 & 4 & 5 & 6 & 7 & 8 & 9 \\
\hline 1. Idade & - & $0,23^{*}$ & 0,03 & 0,02 & $-0,01$ & 0,04 & 0,10 & 0,08 & 0,14 \\
\hline 2. Sexo & $0,23^{*}$ & - & $-0,23^{*}$ & $-0,23^{*}$ & $-0,02$ & $-0,03$ & 0,17 & 0,02 & 0,10 \\
\hline 3. EREEF & 0,03 & $-0,23$ & - & $0,52^{* *}$ & 0,04 & 0,02 & $-0,01$ & $-0,04$ & 0,10 \\
\hline 4. DANVA2 & 0,02 & $-0,23^{*}$ & $0,52^{* *}$ & - & 0,08 & $-0,08$ & $-0,09$ & $-0,08$ & 0,10 \\
\hline 5. CES-D & $-0,01$ & $-0,02$ & 0,04 & 0,08 & - & $-0,70^{\star *}$ & $-0,65^{\star *}$ & $-0,37^{\star *}$ & $-0,17$ \\
\hline 6. LSA & 0,04 & $-0,03$ & 0,02 & $-0,08$ & $-0,70^{* *}$ & - & $0,75^{\star *}$ & $0,34^{* *}$ & $0,23^{*}$ \\
\hline 7. PANAS & 0,10 & 0,17 & $-0,01$ & $-0,09$ & $-0,65^{\star *}$ & $0,75^{\star *}$ & - & $0,38^{* *}$ & $0,22^{*}$ \\
\hline 8. PBIMAT & 0,08 & 0,02 & $-0,04$ & $-0,08$ & $-0,37^{\star *}$ & $0,34^{* *}$ & $0,38^{* *}$ & - & $0,30^{* *}$ \\
\hline 9. PBIPAT & 0,14 & 0,10 & 0,10 & 0,10 & $-0,17$ & $0,23^{*}$ & $0,22^{*}$ & $0,30^{* *}$ & - \\
\hline
\end{tabular}

EREEF - A Escala de Reconhecimento de Expressões Faciais; DANVA2 - Diagnostic Analysis of Nonverbal Accuracy Scale; CES-D Escala de Sintomas de Depressão do CES; LSA - Índice de Satisfação com a Vida; PANAS - Escala de Afetos Positivos e Afetos Negativos. PBIMAT Parental Bonding Instrument - Materno, PBIPAT Parental Bonding Instrument - Paterno.

Participantes com maior satisfação com a vida, tal como avaliado pelo LSA tenderam a apresentar maior presença de afetos positivos, tal como avaliado pela PANAS. De fato, essa foi a mais forte correlação observada no estudo $(0,74)$, indicando que LSA e PANAS compartilham aproximadamente $55 \%$ de sua variância. Esses resultados são compatíveis com a literatura.

Corroborando expectativas, foram identificadas correlações inversas entre sintomatologia de depressão (CES-D), satisfação com a vida (LSA) e balanço de afetos positivos e negativos. Participantes com escores maiores na CES-D, ou seja, com mais sintomas de depressão, tenderam a reportar uma menor satisfação com a vida, tal como avaliado pelo LSA, e uma menor presença de afetos positivos, tal como avaliado pela PANAS. De fato, as correlações entre essas medidas foram altas e negativas oscilando entre $-0,65$ e $-0,70$.

As medidas de qualidade de interação entre pais e filhos (PBI Comportamento Materno e PBI Comportamento Paterno) também apresentaram correlações significativas entre si e com outras variáveis como sintomatologia para depressão e bem-estar subjetivo. Por exemplo, participantes que reportaram melhor qualidade de interação com seus pais tenderam a apresentar menos sintomas de depressão.

No presente estudo não foram identificadas correlações significativas entre as medidas de reconhecimento de emoções e as medidas de sintomatologia para depressão e de bem-estar subjetivo e qualidade de interação com os pais. Correlações significativas 
Rodolfo de Castro Ribas Junior, Marcos de Souza Aguiar, Paula Bandeira Dias, Felipe Salvador Grisolia,

Luiz Gonzaga Ribeiro Vasconcelos Rosario, Felipe Costa Pulcherio Lima \& Paulo Koatz Miragaya

eram esperadas. De fato, embora Carton, Kessler e Pape (1999) tenham identificado correlações essas variáveis em um estudo semelhante, pode-se dizer que estudos sobre reconhecimento de emoções em adultos ainda são relativamente escassos.

\section{CONCLUSÕES}

No presente estudo foi possível investigar o reconhecimento de emoções em expressões faciais em uma amostra de adultos brasileiros. Os instrumentos utilizados, inclusive o desenvolvido pelos autores (EREEF) apresentaram fidedignidade adequada para a investigação psicológica.

Mayer, Roberts e Barsade (2009) ponderaram que os estoicos acreditavam que a racionalidade seria superior aos sentimentos e emoções, porque estes últimos seriam pouco previsíveis, tendo pouca serventia para o pensamento racional. Nesse contexto a expressão dos sentimentos estaria ligada à "natureza feminina". Os autores ressaltam que essa tradição parece prevalecer, em alguma medida, no Ocidente. Em função de uma série de fatores como um processo de socialização diferenciado, mulheres tenderiam a ter um maior conhecimento acerca de emoções. Corroborando uma farta literatura disponível, o presente trabalho identificou que as mulheres foram capazes de reconhecer com mais eficiência as emoções em expressões faciais.

Nossos resultados sugerem que as diferenças de gênero não existem no processamento de estímulos visuais emocional, e ilustram a importância de levar essas diferenças em consideração durante as investigações do processamento emocional. Finalmente, essas diferenças de gênero podem ter implicações para a fisiopatologia de transtornos de humor como a depressão.

Em um estudo envolvendo neuroimagens, Kemp, Silberstein, Armstrong e Nathan (2004) identificaram diferenças relacionadas ao sexo no processamento neuronal de estímulos visuais com carga emocional. Homens e mulheres processariam esses estímulos com carga emocional de forma distinta Segundo os autores esses achados teriam implicações para entendimento de variáveis como sexo no entendimento de patologias relacionadas a desordens de humor e depressão. Entretanto, a origem dessas diferenças ainda precisa ser melhor estabelecida.

No presente estudo a correlação encontrada entre a EREEF e o DANVA2, apesar de significativa, foi moderada $(r=0,52)$. Esse resultado era esperado. A
EREEF e o DANVA2 foram desenvolvidos em contextos diferentes e não houve preocupação de parar os instrumentos considerando variáveis com nível de intensidade do estímulo. A utilização de uma escala consagrada internacionalmente poderá facilitar futuras comparações com resultados obtidos em outros estudos internacionais.

O estudo apresenta uma limitação que pode ser minimizada com o desenvolvimento dos estudos: a amostra utilizada foi limitada. O estudo envolveu apenas estudantes universitários e o tamanho da amostra (apesar de suficiente para garantir a potência das análises) não pode ser considerado expressivo. Vale à pena ressaltar, entretanto, que a diferença nas percentagens de participantes do sexo feminino e masculino foi controlada estatisticamente em todas as análises.

É importante destacar que se trata de um estudo preliminar, limitando-se à investigação de quatro emoções (alegria, tristeza, raiva e medo). Considerando a relevância do tema e a relativa escassez de estudos brasileiros, os autores pretendem dar continuidade aos estudos e estimular a participação de outros grupos de pesquisa, com a utilização de instrumentos que avaliem mais emoções.

\section{REFERÊNCIAS}

Alves-Neto, W. C., Guapo, V. G., Graeff, F. G., Deakin, J. F. W., \& Del-Ben, C. M. (2010). Effect of escitalopram on the processing of emotional faces. Brazilian Journal of Medical and Biological Research, 285-289.

Brody, L. R. (1985). Gender differences in emotional development: A review of theories and research. Journal of Personality, 53, 102-149.

Carton, J. S., Kessler, E. A., \& Pape, C. L. (1999).Nonverbal decoding skills and relationship well-being in adults.Journal of Nonverbal Behavior, 23, 91-100.

Cervantes, C. A., \& Callanan, M. A. (1998). Labels and explanations in mother-child emotion talk: Age and gender differentiation. Developmental Psychology, 34, 88-98.

Darwin, C. (1872/1965). The expression of the emotions in man and animals. Chicago: The University of Chicago Press.

Ekman, P. (1993). Facial expression and emotion. American Psychologist, 48(4), 384-392.

Ekman, P. (2012). Respect in a Pinch. Science, 337, 1173-1173.

Ekman, P. \& Cordaro, D. (2011). WhatisMeantbyCallingEmotions Basic. Emotion Review, 3, 364-370.

Ekman, P. \& Friesen, W. V. (1971). Constant sacross cultures in face and emotion. Journal of Personality and Social Psychology, $17,124-124$. 
Ekman, P. \& Oster, H. (1979). Facial expressions of emotion. Annual Review of Psychology, 30, 527-554.

Goodfellow, S., \& Nowicki, S. (2009). Social Adjustment, Academic Adjustment, and the Ability to Identify Emotion in Facial Expressions of 7-Year-Old Children. Journal of Genetic Psychology, 170, 234-243.

Hauck, S., Schestatsky, S., Terra, L., Knijnik, L., Sanchez, P., \& Ceitlin, L. H. F. (2006). Adaptação transcultural para o português brasileiro do Parental Bonding Instrument (PBI). Revista de Psiquiatria do Rio Grande do Sul, 28, 162-168.

Hoehl, S., \& Striano, T. (2008). Neural Processing of Eye Gaze and Threat-Related Emotional Facial Expressions in Infancy.Child Development, 79, 1752-1760.

Izard, C. E. (2001). Emotional Intelligence or Adaptive Emotions? Emotion, 1, 249-257.

Izard, C. E. (2009). Emotion Theory and Research: Highlights, Unanswered Questions, and Emerging Issues. Annual Review of Psychology, 60, 1-25.

Izard, C., Fine, S., Schultz, D., Mostow, A., Ackerman, B., \& Youngstrom, E. (2001).Emotion knowledge as a predictor of social behavior and academic competence in children at risk. Psychological Science., 12, 18-23.

Izard, C., Stark, K., Trentacosta, C., \& Schultz, D. (2008). Beyond Emotion Regulation: Emotion Utilization and Adaptive Functioning. Child Development Perspectives, 2, 156-163.

Johnson, D. R. (2009). Emotional Attention Set-Shifting and Its Relationship to Anxiety and Emotion Regulation. Emotion, 9, 681-690.

Kemp, A. H., Silberstein R. B., Armstrong, S. M., Nathan, P. J. (2004). Gender differences in the cortical electrophysiological processing of visual emotional stimuli. Neuroimage, 16, 632646.

Kring, A. M., \& Gordon, A. H. (1998). Sex differences in emotion: Expression, experience, and physiology. Journal of Personality and Social Psychology, 74, 686-703.

Mayer, J. D., Roberts, R. D., \& Barsade, S. G. (2008). Human abilities: Emotional intelligence. Annual Review of Psychology, 59, 507-536.

Mayer, J. D., Salovey, P., \& Caruso, D. R. (2008). Emotional intelligence - New ability or eclectic traits? American Psychologist, 63, 503-517.

McClure, E, \& Nowicki, S. (2001). Associations between social anxiety and Nonverbal processing skill in preadolescent boys and girls. Journal of Nonverbal Behavior, 25, 3-19.

McClure, E. B. (2000). A meta-analytic review of sex differences in facial expression processing and their development in infants, children, and adolescents. [Article; Proceedings Paper]. Psychological Bulletin, 126, 424-453.

Montirosso, R., Peverelli, M., Frigerio, E., Crespi, M., \& Borgatti, R. (2010).The Development of Dynamic Facial Expression Recognition at Different Intensities in 4-to 18-Year-Olds. Social Development, 19, 71-92.
Mufson, L., \& Nowicki Jr, S. (1991). Factors affecting the accuracyof facial affect recognition. The Journal of Social Psychology, $131,815-822$.

Neves, M. d. C. L. d., Tremeau, F., Nicolato, R., Lauar, H., Romano-Silva, M. A., \& Correa, H. (2011). Facial emotion recognition deficits in relatives of children with autism are not associated with 5HTTLPR. Revista Brasileira de Psicologia, 33, 0-0.

Nowicki Jr, S., \& Carton, E. (1997). The Relation of Nonverbal Processing Ability of Faces and Voices and Children's Feelings of Depression and Competence. Journal of Genetic Psychology, 158, 357-363.

Nowicki Jr, S., \&Carton, J. (1993). The measurement of emotional intensity from facial expressions. The Journal of Social Psychology, 133, 749-750.

Nowicki Jr, S., \& Duke, M. P. (1994). Individual differences in the nonverbal communication of affect: The diagnostic analysis of non verbal accuracy scale. Journal of Nonverbal Behavior, $18,9-35$.

Nowicki Jr, S., Glanville, D., \& Demertzis, A. (1998). A test of the ability to recognize emotion in the facial expressions of African American adults. Journal of Black Psychology, 24, 335-350.

Pears, K. C., \& Fisher, P. A. (2005). Emotion understanding and theory of mind among maltreated children in foster care: Evidence of deficits. Development and Psychopathology, 17, 4765 .

Propper, C., \& Moore, G. A. (2006). The influence of parenting on infant emotionality: A multi-level psychobiological perspective. Developmental Review, 26, 427-460.

Radloff, L. S. (1977). The CES-D Scale. Applied Psychological Measurement, 1, 385-401.

Robins, R. W., Gosling, S. D., \& Craik, K. H. (1999). An empirical analysis of trends in psychology. American Psychologist, 54, 117-128.

Southam-Gerow, M. A., \& Kendall, P. C. (2002). Emotion regulation and understanding - Implications for child psychopathology and therapy.Clinical Psychology Review, 22, 189-222.

van Beek, Y., \& Dubas, J. S. (2008). Age and gender differences in decoding basic and non-basic facial expressions in late childhood and early adolescence. Journal of Nonverbal Behavior, 32, 37-52.

Vigil, J. M. (2009). A socio-relational framework of sex differrences in the expression of emotion. Behavioral and Brain Sciences, 32, 375-428.

Watson, D., Clark, L. A., \& Tellegen, A. (1988). Development and validation of brief measures of positive and negative affect - the PANAS scales. Journal of Personality and Social Psychology, 54, 1063-1070.

Weber, E. U., \& Johnson, E. J. (2009). Mindful Judgment and Decision Making. Annual Review of Psychology, 60, 53-85. 
Wickline, V. B., Bailey, W., \& Nowicki, S. (2009). Cultural InGroup Advantage: Emotion Recognition in African American and European American Faces and Voices. Journal of Genetic Psychology, 170, 5-28.

Wong, Y. L. I. (2000). Measurement properties of the Center for Epidemiologic Studies - Depression Scale in a homeless population. Psychological Assessment, 12, 69-76.

Recebido em 23/11/2011 Última revisão em 11/07/2013 Aceito em 13/08/2013 